\title{
Children with autism spectrum disorders and selective mutism
}

This article was published in the following Dove Press journal:

Neuropsychiatric Disease and Treatment

\author{
Hanna Steffenburg \\ Suzanne Steffenburg \\ Christopher Gillberg \\ Eva Billstedt \\ Gillberg Neuropsychiatry Centre, \\ Institute of Neuroscience \\ and Physiology, University \\ of Gothenburg, Sahlgrenska \\ Academy, Gothenburg, Sweden
}

\begin{abstract}
Background: It has been suggested that autism spectrum disorder (ASD) might be a "comorbid" condition in selective mutism (SM).

Methods: In this retrospective study, we examined medical records of children with SM diagnosis ( $\mathrm{n}=97)$ at a medical center specializing in assessment of ASD.

Results: Mean age for onset of SM symptoms was 4.5 years and mean age at SM diagnosis was 8.8 years. SM was more common among girls (boy:girl ratio=2.7:1). We found that $63 \%$ of the study group had an ASD (no gender difference). The SM group with combined ASD had later onset of symptoms, higher age at diagnosis, more often a history of speech delay, and a higher proportion of borderline IQ or intellectual disability.
\end{abstract}

Conclusion: The results highlight the risk of overlap between ASD and SM.

Keywords: selective mutism, autism spectrum disorders, Asperger syndrome, autistic disorder

\section{Introduction}

Selective mutism (SM) is described in the Diagnostic and Statistical Manual of Mental Disorders, Fourth edition (DSM-IV) and Fifth edition (DSM-5) as a rare anxiety disorder, characterized by a consistent failure to speak in specific social situations in which there is an expectation of speaking, despite speaking in other situations ${ }^{1,2}$ (Americal Psychological Association 1994; 2013). In 1877, Kussmaul described SM and he used the term aphasia voluntaria to underline the awareness and the free will not to speak. ${ }^{3}$ In 1934, Tramer ${ }^{3}$ introduced the concept of elective mutism that continued emphasizing the voluntariness in not speaking in certain situations. Throughout its history, SM has been described as an oppositional trait with an unwillingness to speak rather than a single diagnosis. ${ }^{3}$ In the publication of DSM-IV, ${ }^{1}$ the diagnosis of SM was included, which underlines the lack of speech in certain situations. The shift broadened the diagnosis phenomenology, from a voluntarily controlled and defiant behavior to a pattern of reaction in response to an overwhelming or threatening context change. ${ }^{4}$ The failure to speak cannot be explained by lack of language skills. The duration of disturbance should be at least 1 month. The disorder should not be categorized as a communication disorder, pervasive developmental disorder (PDD), schizophrenia, or other psychotic disorder. ${ }^{1,2}$ In the DSM-5, SM is categorized under the category of Anxiety Disorders, and this marks an increased focus on supposed underlying social anxiety problems.

Communication patterns in SM vary from total absence of speech in almost all situations to lack of speech in only certain situations. The most common situations for the appearance of SM symptoms are in school, where the teacher is the person that the child is least likely to talk to. ${ }^{5}$ Sometimes the child can talk to select students, sometimes to nobody at all. Commonly, the child speaks normally at home with the
Correspondence: Eva Billstedt Gillberg Neuropsychiatry Centre, University of Gothenburg, Kungsgatan 12, Gothenburg 4II 19, Sweden

$\mathrm{Tel}+463$ I 3425980

Fax +46 3I 3425979

Email eva.billstedt@gnc.gu.se 
family, but the opposite situation, where the child speaks at school but not at home, has also been described. ${ }^{6}$

Onset of SM symptoms is reported to be 2.7-4.1 years. ${ }^{4}$ Usually, the symptoms are not noticed until the child starts primary school, which might be explained by the increased social demand and increased demands for performance, making the symptoms more obvious. ${ }^{3}$ The long interval between symptom onset and detection of problems is probably explained by the fact that children often speak without any problem in the home environment.

The reported prevalence rates vary between $0.2 \%$ and $0.8 \%{ }^{4,7-9} \mathrm{SM}$ is reported to be more common among girls than boys, with an estimated girl:boy ratio varying between 2.6:1 and 1.5:1. ${ }^{3} \mathrm{SM}$ is described as a condition in children but can also be recognized in adults; however, the symptoms are more often categorized as social phobia, ${ }^{3}$ under the anxiety disorders umbrella. A few longitudinal studies of SM have been performed and the persistence of symptoms varies in the literature, from months to several years. There is usually remaining shyness; social anxiety; ${ }^{3}$ and lack of self-confidence, independence, achievement, and social communication abilities into teenage and adult life. ${ }^{6,10}$

In addition to anxiety, other associated symptoms/comorbidities have also been reported, such as speech and language problems, ${ }^{11,12}$ obsessive compulsive disorder, ${ }^{13}$ Fragile $\mathrm{X}$ syndrome, ${ }^{6}$ attention deficit hyperactivity disorder (ADHD) ${ }^{4}$ depression, panic disorder, disassociate disorders, and other somatic complaints. ${ }^{3}$ Kristensen reported an association between SM and developmental disorders relating to communication, learning, motor skills in a study of 54 children with SM. She found that $7 \%$ of the participants also fulfilled the criteria for Asperger syndrome (AS), and an additional 7\% met the criteria for mild intellectual disability (ID). ${ }^{12}$ Anderson and Thomsen ${ }^{14}$ found in a group of 37 children with SM that 4 of them had AS. Furthermore, autism spectrum disorder (ASD) has been found to occur more frequently in SM families. ${ }^{14}$

The purpose of the present study was to examine the prevalence of ASD in a relatively large clinical sample of children with SM who were referred to a center specializing in the assessment of neurodevelopmental disorders. Furthermore, a study on joint attention, a specific aspect of social communication, reported fewer episodes of joint attention in the SM group than in comparison group. ${ }^{15}$ More girls than boys are affected by SM; ${ }^{3}$ however, if that is the case in children with comorbid SM and ASD is not clear. A second aim was therefore to examine possible gender differences with regard to presence of ASD.

\section{Participants and methods Procedure}

All individuals at the Child Neuropsychiatry Clinic (CNC) in Gothenburg who had received a DSM-IV diagnosis of SM between 2003 and 2014 were eligible for participation in the study. CNC is a regional clinic in Gothenburg, West Sweden, focusing on the assessment of neurodevelopmental disorders (autism, ADHD, Tourette syndrome, etc), and at that period also on the assessment of SM, in children and adolescents. All data were retrieved from medical records including the diagnosis, except for in some cases where there was an ASD diagnostic re-evaluation based on the medical records at the time of the study, by the second author SZ, who was the clinical doctor for a large proportion of the study group. The time period was chosen to coincide with the introduction of a computerized medical record system at the CNC in 2003, making data retrieval easier.

\section{Participants}

One hundred and six individuals were diagnosed as having SM during the selected time period at the CNC. Nine individuals ( 4 girls and 5 boys) were excluded because there was some uncertainty about diagnosis or because the medical records contained too scanty information, leaving a total of 97 individuals ( 71 girls and 26 boys) who were included in the study. The age at assessment in the $\mathrm{CNC}$ ranged from 4 to 18 years (median $=8$ years).

\section{The clinical/diagnostic assessment}

The clinical/diagnostic assessments had been performed by a team that included a medical doctor/child psychiatrist and a clinical psychologist at $\mathrm{CNC}$, both with extensive experience in the field of SM and ASD, including diagnostic work-up. The diagnoses were based on all the available information provided by the files, the clinical interview, and the clinical impression according to the LEAD-principle (Longitudinal, Expert, All, Data). ${ }^{16}$ All individuals were examined individually, and at least 1 parent was interviewed. The interview always covered language development, onset of SM symptoms, age at diagnosis, and whether or not the child had been exposed to a non-Swedish language environment at home. The clinical assessment procedure included instruments regularly used in clinical practice, such as DSM-IV checklists, parent/teacher questionnaires (the 5-15 questionnaire ${ }^{17}$ ), and The Autism Spectrum Screening Questionnaire ${ }^{18}$ in most cases. The choice of instrument was dependent on the complexity of the condition (the more complex the clinical picture, the more extended 
assessment) or if the child was referred to the clinic for second opinion evaluation (meaning that several instruments had already been used at the referral clinic). However, these instruments were not used as a final decision for diagnosis. The doctor made the final diagnostic decision after consultation with the clinical psychologist. The diagnoses of SM and autistic disorder (AD) were based on the DSM-IV criteria. ${ }^{1}$ PDD not otherwise specified (PDD-NOS)/atypical autism was diagnosed when DSM-IV criteria for $\mathrm{AD}$ were met. For the diagnosis of AS, the Gillberg and Gillberg criteria ${ }^{14}$ were applied. The term ASD was used as an umbrella term to include children with AD, PDD-NOS/atypical autism, and AS. Children with autistic features (meeting 2 or $3 \mathrm{AD}$ criteria) were described as having autistic features but they were not included in the ASD-group. A combination of ASD and SM diagnosis was given when the symptoms could not be explained by one of the separate conditions alone; for example, when the child was reported to have absence of speech in certain situations but not in others in combination with non-verbal communication deficits and extreme adherence to routines.

\section{Assessment of cognitive function}

About half the study group had been cognitively assessed with an IQ test (Wechsler Preschool or Primary Scale of Intelligence, Wechsler Intelligence Scale for Children, Wechsler Adult Scale of Intelligence ${ }^{19-21}$ ) or, in children with a mental age below what is required in the Wechsler scale test, a developmental test (Griffiths' Developmental Scales I and/or $\mathrm{II}^{22}$ ) was used. In some cases an evaluation of the cognitive function was based on the overall clinical impression of the child. All assessments (including evaluation) of cognitive or developmental function had been performed by a licensed psychologist either at the referral clinic or at the CNC. Cognitive level group status was assigned in the following manner: Average Intellectual Functioning $(\mathrm{AIF})=\mathrm{IQ}>84$, Borderline Intellectual Functioning (BIF) IQ 70-84, and ID=IQ $<70$.

\section{Statistical analysis}

Descriptive analyses were used to obtain information on means and SD for continuous variables, and frequencies and percentages for categorical variables. The non-parametric test Chi-squared was applied for analysis of nominal data and MannWhitney $U$-test was applied for analysis of interval data.

\section{Ethical approval}

The study was approved by the Medical Ethical Committee of the University of Gothenburg. This is an anonymized baseline register clinical audit study and patient consent was not required at this stage, but for the ongoing follow-up study, there is ethics approval with written informed consent. All procedures performed in the study were in accordance with the ethical standards of the institutional research committee and with the 1964 Helsinki declaration and its later amendments or comparable ethical standards.

\section{Results \\ Comorbid ASD in the SM study group}

Of the 97 individuals with SM, 63\% ( $n=61)$ had ASD, hereinafter referred to as the SM+ASD group. Of these, 28 (29\%) had AD, 29 (30\%) had atypical autism/PDD-NOS, and $4(4 \%)$ had AS. A further 20\% ( $n=19)$ had autistic features that were "subclinical", but, nevertheless, sufficiently marked to have an impact on everyday life. No ASD symptoms at all were found in $17 \%(n=17)$. The group with a few autistic features, but not enough for a formal ASD diagnosis, and the group with no ASD symptoms were collapsed and are referred to as the SM-ASD group $(n=36,37 \%)$.

\section{Delayed or abnormal speech development}

For $17 \%(n=14)$, no information about speech development was recorded. About 1 in $4(n=22 / 83,27 \%)$ of the total study group was reported in medical records to have had delayed or abnormal speech development (Table 1). There was a trend that the SM+ASD group $(n=17 / 51,33 \%)$ had more delayed/abnormal speech development than the SM-ASD group ( $\mathrm{n}=5 / 32,16 \%$ ) but this difference was not significant $(p=0.0752)$.

\section{Onset of SM symptoms}

The mean age of onset of symptoms ( $\mathrm{n}=92$, missing information in 5 cases) was 4.5 years and the range was $1-16$ years of age. Only 4 cases were reported to have symptom onset after 10 years of age. The onset of symptoms was very often reported to be related to start of preschool (age 2-4 years) or when the child changed preschool group, particularly from a smaller to a larger group or at start of school (in Sweden about 7 years of age). Mean age for onset of SM symptoms in the $\mathrm{SM}+\mathrm{ASD}$ group was reported to be 5.0 years $(\mathrm{SD}=2.7)$ compared to 3.7 years $(\mathrm{SD}=1.9)$ in the "SM Only" group ( $U=694, p=0.011)$.

\section{Age at SM diagnosis}

The mean age at SM diagnosis in the total study group was 8.8 years $(\mathrm{SD}=3.6)$, with an age range between 4 and 18 years 
Table I Clinical characteristics of 97 children with SM, SM+ASD, or SM-ASD

\begin{tabular}{|c|c|c|c|c|}
\hline & $\begin{array}{l}\text { Total SM, } \\
\text { N=97, n (\%) }\end{array}$ & $\begin{array}{l}\text { SM-ASD, } \\
n=36, \mathrm{n}(\%)\end{array}$ & $\begin{array}{l}\text { SM+ASD, } \\
n=6 I, n(\%)\end{array}$ & $\begin{array}{l}\text { Comparison between } \\
\text { SM-ASD and } \\
\text { SM+ASD, } p \text {-value }\end{array}$ \\
\hline Girl:boy ratio & $71: 26$ & $43: 18$ & $28: 8$ & 434 \\
\hline Delayed speech development $(n=83)$ & $22(27)$ & $5(16)$ & $17(33)$ & 0.121 \\
\hline Age at onset of SM symptoms, mean $(95 \% \mathrm{Cl})(\mathrm{n}=92)$ & $4.5(4.0-5.0)$ & $3.6(3.0-4.2)$ & $5.0(4.3-5.8)$ & 0.006 \\
\hline \multicolumn{5}{|l|}{ Intellectual function } \\
\hline Average or above average IQ $(\geq 85)$ & $60(62)$ & $33(92)$ & $27(44)$ & $<0.001$ \\
\hline Borderline IQ (7I-84) & $21(22)$ & $2(6)$ & $19(31)$ & 0.004 \\
\hline Intellectual disability $(\leq 70)$ & $16(16)$ & $I(3)$ & $15(25)$ & 0.004 \\
\hline \multicolumn{5}{|l|}{ ASD } \\
\hline Autistic disorder & $28(29)$ & 0 & $28(46)$ & $<0.001$ \\
\hline Asperger syndrome & $4(4)$ & 0 & $4(7)$ & 0.117 \\
\hline PDD-NOS & $29(30)$ & 0 & $29(48)$ & $<0.001$ \\
\hline Autistic traits (no diagnosis) & $19(20)$ & $19(53)$ & $0(0)$ & $<0.001$ \\
\hline Context of symptoms outside home & $97(100)$ & $36(100)$ & $61(100)$ & 1.0 \\
\hline Context of symptoms in home & $5(5)$ & I (3) & $4(7)$ & 0.377 \\
\hline Non-Swedish language home environment & $26(27)$ & $14(39)$ & $12(20)$ & 0.057 \\
\hline Age at diagnosis of SM, mean $(95 \% \mathrm{Cl})$ & $8.8(8.0-9.5)$ & $6.9(5.8-7.9)$ & $9.9(9.0-10.8)$ & $<0.001$ \\
\hline
\end{tabular}

Abbreviations: ASD, autism spectrum disorder; IQ, intellectual quotient; NOS, not otherwise specified; PDD, pervasive developmental disorder; SM, selective mutism.

of age. Mean age of SM diagnosis in the SM+ASD group was 9.9 years $(\mathrm{SD}=3.4)$ and in the "SM Only" group 6.9 years $(\mathrm{SD}=3.1)(U=501, p<0.001)$.

\section{Non-Swedish-speaking home environment}

Twenty-seven percent (26/97) of the children were living in a home where more than one language was spoken, and this was more common among males than females $\left(\chi^{2}=4.35\right.$, $p<0.05)$. One in 5 in the SM+ASD group had such a home environment compared with almost 2 in 5 in the "SM Only" group $\left(X^{2}[1, \mathrm{~N}=97, p=0.057]\right)$.

\section{Clinical SM characteristics at assessment}

SM symptoms manifested outside the home in all cases $(100 \%)$. Five percent $(n=5)$ had symptoms of SM in the home as well. These children were described as extremely shy and avoiding contact with others, alternatively not talking to a specific member of the family. Four of these had SM+ASD.

\section{General cognitive function}

In the total study group, $62 \%(\mathrm{n}=60)$ had AIF, $22 \%$ had BIF, and $16 \%$ had ID. There was a significantly lower cognitive function in the SM+ASD group compared with the SM-ASD group ( $U=572, p<0.001)$. In the SM+ASD group, $27(44 \%)$ had AIF, 19 (31\%) had BIF, and 15 children (25\%) had ID.
In the SM+ASD group, we found that 33 children ( $92 \%)$ had AIF, 2 (6\%) had BIF, and 1 had ID (3\%) $(p=0.001)$.

\section{Gender difference}

There were 71 girls and 26 boys (girl:boy ratio=2.7:1 $[p<0.001])$ in the total study group. No significant gender ratio difference was found between the SM+ASD group (43 girls, 18 boys, girl:boy ratio=2.4:1) compared with the SM-ASD group (28 girls, 8 boys, girl:boy ratio=3.5:1, Table 1$)$. We found no gender differences regarding speech development, delay/ abnormal development, onset of symptoms, age of diagnosis, or cognitive functioning. The only gender difference found was that more boys than girls were living in families where Swedish was not the first language.

\section{Discussion}

In this study of a clinical group of children who were diagnosed with SM and assessed at a center for neurodevelopmental disorders, $63 \%$ also met criteria for ASD. A further $20 \%$ had subclinical autistic traits. This is a significantly higher prevalence than found in Kristensen's study, ${ }^{12}$ or in the Anderson et al study ${ }^{14}$ which are the only 2 systematic clinical studies, to our knowledge, that set out to study the prevalence of neurodevelopmental disorders in SM. Kristensen found that $7 \%$ of her group had AS, which is similar to the rate reported by Anderson et al. ${ }^{14}$ Our much higher rate of children with ASD in the present study most likely reflects 
that the participants were referred to $\mathrm{CNC}$, a clinic that is well known for their expertise in ASD. This means that our results correspond well with those children who have an additional neurodevelopmental disorder and probably not with entire SM population. Additionally, this is a retrospective chart review study with the limitations that such studies entail. However, the professionals responsible for the chart records were extremely experienced clinical experts in the field of autism and general child neuropsychiatry, and their information entries in the medical records therefore were quite homogeneous. The reason why the individuals with ASD had not been previously "properly" diagnosed as suffering from ASD cannot be specified with certainty, but we believe that the combination of 1) the general tendency for clinicians to only provide one major "overarching" diagnosis and 2) the very obvious (indeed often dramatic) symptom of muteness had led to a primary diagnosis of "only" SM. Also, up until very recently, double diagnosis has not been strongly supported in the guidelines of the diagnostic manuals, such as the DSM. Both SM and ASD have been regarded by many (both clinicians and researchers) as homogeneous, indeed mutually exclusive, disorders with little or no comorbidity. We would argue that our findings are important in that they point to the need toward always looking out for symptoms of autism in cases with SM, and to consider the possibility that the "symptom" of SM might not be extremely rare in autism.

It has been suggested that the anxiety experienced by children with SM makes them refuse speech because they are "frozen with fear". ${ }^{24}$ Our study group with SM+ASD was more often described as demonstrating lack of social skills/social interest, and that refusal to speak reflected a stubborn refusal rather than shyness. There might be subgroups within the SM patient group and those who were assessed at our clinic are particularly the subgroup with SM and a comorbid ASD.

It is probably important for the group of individuals who meet the criteria for both SM and ASD that both diagnoses be considered. It is likely that the principles for intervention in ASD (eg, preparation for new events, visual support for daily structure, and to make daily activities predictable) and the knowledge of communication problems seen in ASD (that it always includes impairments in the social use of language) are beneficial for the comorbid SM+ASD group. However, it is not known whether treatment directed at SM should be differently designed when autism is concurrent.

The mean age of SM symptom onset was 3.7 years in the SM-ASD group, and 18 months later in the SM+ASD group. Onset of symptoms in the SM-ASD group is consistent with previous research where an onset between 2 and 5 years of age has been reported. ${ }^{25}$ Our finding of a considerably later onset of SM symptoms in the SM+ASD group could, perhaps, be explained by SM being "due to" autism or SM being overshadowed by other ASD symptoms. Overall, the findings indicate an early age of onset of SM, estimated to be when the child is in preschool, aged 2-5 years.

Early onset of symptoms does not lead directly to early diagnosis. The mean age for SM diagnosis was about 3 years after SM symptom onset and almost 5 years later for the combined SM+ASD. This implies that several years of intervention and treatment are missed, which are important years in a child's development of social communication and interaction. It is also important to highlight that the age range for the diagnosis of SM ranged from 4 to 18 years. For some children, it may be more than 4 years between onset of symptoms and diagnosis time.

We found an uneven girl:boy ratio, with more girls than boys presenting the condition. This was also reflected in the $\mathrm{SM}+\mathrm{ASD}$ group. Compared with other neurodevelopmental/ neuropsychiatric disorders, this higher prevalence of SM in girls is perhaps somewhat surprising. It is possible that the designed diagnostic criteria for SM could fit a more typical girls "prototype" with introverted symptoms, such as quiet and restrained behavior. However, our results are in line with other studies that show all anxiety disorders occur more frequently among females than males. ${ }^{25}$

All reported SM symptoms were expressed outside home, which is to be expected since the social setting described in the criteria is outside home (school). Interestingly, there was a small subgroup (only about $5 \%$ of the total group) who also presented major SM symptoms at home, in familiar settings toward a family member, and that all except 1 of these children also had ASD.

Immigration and exposure to several languages during the early years has been reported to be a possible risk factor for developing SM. ${ }^{27}$ We could not confirm a higher than average rate of this type of background in the SM group compared with the general population of children under 18 years of age (27\% in the SM group versus $30 \%$ in children under 18 years of age in the general population, Statistics Sweden ${ }^{28}$ ). However, there was a trend that the SM+ASD group more often had a bilingual background compared with the SM+ASD group, indicating that in the latter group, additional neurodevelopmental factors might play an etiological role in the development of SM.

The level of cognitive function was average in more than half of the study group but more than one-third of the 
study group had a borderline IQ or an ID. Prevalence rates for ID vary between $0.5 \%$ and $2 \% 0^{29-31}$ and the estimated rate for borderline IQ is $10 \%-15 \%$ in Swedish primary school children. ${ }^{31}$ Our findings suggest that low cognitive function may be more common in the SM group. It has been suggested that children with lower intelligence or learning problems perceive school as more difficult, and because of that, they are less likely to engage in speech ${ }^{24}$ and it is probable that low cognitive ability, combined with difficulties, such as autism, can affect the development of SM. On the other hand, the lower cognitive ability might be due to having professionals administer the test, which is the standard practice. In a study by Klein et al, children with SM scored higher on parents-administered test than on professional-administered test measuring receptive and expressive language, albeit not on narrative comprehension test. ${ }^{33}$

Our results not only show an overlap between SM and ASD but also IDs. A concept that might better capture this overlap is Early Symptomatic Syndromes Eliciting Neurodevelopmental Clinical Examinations (ESSENCE). The concept was founded by Gillberg ${ }^{34}$ and aims at the whole group of early-onset neurodeveloping problems, regardless of diagnosis, and emphasizes that these problems often overlap with each other and that there is a need for clinicians to be alert aware of this. Because of the overlap between symptoms, patients are sometimes diagnosed with 1 or 2 diagnoses at one point and a third at a later stage. Disorders included in the ESSENCE concept include, but are not limited to, ASD, ADHD, IDs, language disorders, and motor disorders. An ESSENCE approach would mean that occurring SM symptoms constitutes a marker to draw attention to other neurodevelopmental abnormalities, such as autism or IDs.

\section{Strength and limitations}

The study is based on retrospective chart reviews, which implies some uncertainty as to the quality of the data obtained at the time for diagnostic assessment. All participants were diagnosed (or re-evaluated) at one specific clinic, meaning that the data were assessed, analyzed, and interpreted by the same clinical group of professionals, increasing the inter-rater reliability. The study group was a clinic-based cohort and the representativeness of the cases cannot be confirmed.

In comparison to other clinical studies, this study has a special position in terms of number of participants, which makes the study unique. Previous studies have included SM groups of 37-54 individuals. ${ }^{11,13}$ The largest cohort ever included 100 participants, ${ }^{10}$ but there was no focus on ASD in that study.

\section{Disclosure}

The authors report no conflicts of interest in this work.

\section{References}

1. American Psychiatric Association. Diagnostic and Statistical Manual of Mental Disorders. 4th ed. Text revision. Washington, DC, USA: APA; 2000.

2. American Psychiatric Association. Diagnostic and Statistical Manual of Mental Disorders. 5th ed. Washington, DC, USA: APA; 2013.

3. Sharp W, Sherman C, Gross A. Selective mutism and anxiety: a review of the current conceptualization of the disorder. J Anxiety Disord. 2007; 21(4):568-579.

4. Viana G, Beidel D, Rabian B. Selective mutism: a review and integration of the last 15 years. Clin Psychol Rev. 2009;29(1):57-67.

5. Hua A, Major N. Selective mutism. Curr Opin Pediatr. 2016;28(1): 114-120.

6. Sharkey L, McNicholas F. More than 100 years of silence, elective mutism: a review of the literature. Eur Child Adolesc Psychiatry. 2008; 17(5):255-263.

7. Kopp S, Gillberg G. Selective mutism: a population-based study: a research note. J Child Psychol Psychiatry. 1997;38(2):257-262.

8. Bergman R, Piacentini J, McCracken J. Prevalence and description of selective mutism in a school-based sample. J Am Acad Child Adolesc Psychiatry. 2002;41(8):938-946.

9. Elizur Y, Perednik R. Prevalence and description of selective mutism in immigrant and native families: a controlled study. $J$ Am Acad Child Adolesc Psychiatry. 2003;42(12):1451-1459.

10. Remschmidt H, Poller M, Herpertz-Dahlmann B, Hennighausen K, Gutenbrunner C. A follow-up study of 45 patients with elective mutism. Eur Arch Psychiatry Clin Neurosci. 2001;251(6):284-296.

11. Steinhausen HC, Juzi C. Elective mutism: an analysis of 100 cases. J Am Acad Child Adolesc Psychiatry. 1996;35(5):265-288.

12. Kristensen H. Selective mutism and comorbidity with developmental disorder/delay, anxiety disorder and elimination disorder. J Am Acad Child Adolesc Psychiatry. 2000;39(2):249-256.

13. Wong P. Selective mutism: a review of etiology, comorbidities and treatment. Psychiatry Edgemont. 2010;7(3):23-31.

14. Anderson C, Thomsen P. Electively mute children: an analysis of 37 Danish cases. Nord J Psychiatry. 1998;52(3):231-238.

15. Nowakowski ME, Tasker SL, Cunningham CE, et al. Joint attention in parent-child dyads involving children with selective mutism: a comparison between anxious and typically developing children. Child Psychiatry Hum Dev. 2011;42(1):78-92.

16. Spitzer RL. Psychiatric diagnosis: are clinicians still necessary? Compr Psychiatry. 1983;24(5):399-411.

17. Kadesjö B, Janols LO, Korkman M, et al. The FTF (Five to Fifteen): the development of a parent questionnaire for the assessment of ADHD and comorbid conditions. Eur Child Adolesc Psychiatry. 2004; 13(3):3-13.

18. Ehlers S, Gillberg C, Wing L. A screening questionnaire for Asperger syndrome and other high-functioning autism spectrum disorders in school age children. J Autism Dev Disord. 1999;29(2):129-141.

19. Gillberg IC, Gillberg C. Asperger syndrome-some epidemiological considerations: a research note. J Child Psychol Psychiatr. 1998;30 (4):631-638.

20. Wechsler D. Wechsler Preschool and Primary Scale of Intelligence. 3rd ed. Stockholm, Sweden: Psykologiförlaget; 2005.

21. Wechsler D. Wechsler Intelligence Scale for Children. 4th ed. Stockholm, Sweden: Psykologiförlaget; 2007.

22. Wechsler D. Wechsler Adult Intelligence Scale-Third edition. Swedish version. Stockholm, Sweden: Psykologiförlaget; 2003. 
23. Alin-Akerman B, Nordberg L. Griffiths Developmental Scales I and II. Stockholm, Sweden: Psykologiforlaget AB; 1980.

24. Yeganeh R, Beidel DC, Turner SM, Pina AA, Silverman WK. Clinical distinctions between selective mutism and social phobia: an investigation of childhood psychopathology. J Am Acad Child Adolesc Psychiatry. 2003;42(9):1069-1075.

25. Muris P, Ollendick TH. Children who are anxious in silence: a review on selective mutism, the new anxiety disorder in DSM-5. Clin Child Fam Psychol Review. 2015;18(2):151-169.

26. Beesdo K, Knappe S, Pine DS. Anxiety and anxiety disorders in children and adolescents: developmental issues and implications for DSM-V. Psychiatr Clin North Am. 2009;32(3):483-524.

27. Manassis K, Tannock R, Garland J, Minde K, McInnes A, Clark S. The sounds of silence: language, cognition, and anxiety in selective mutism. J Am Acad Child Adolesc Psychiatry. 2007;46(9):1187-1195.

28. Statistics Sweden. Vart femte barn har en utländsk bakgrund. $\mathrm{Nr}$ 2013:2, Stockholm, Sweden: Statistics Sweden; 2013.

29. Hagberg B, Hagberg G, Lewerth A, Lindberg U. Mild mental retardation in Swedish school children. Prevalence. Acta Paediatr Scand. 1981;70(4):441-444.
30. Fernell E. Mild mental retardation in schoolchildren in a Swedish suburban municipality: prevalence and diagnostic aspects. Acta Paediatrica. 1996;85(5):584-588.

31. Landgren M, Pettersson R, Kjellman B, Gillberg C. ADHD, DAMP and other neurodevelopmental/neuropsychiatric disorders in 6-year-old children: epidemiology and co-morbidity. Dev Med Child Neurology. 1996;38(10):891-906.

32. Gillberg C. Neuropsykiatriska funktionshinder hos barn och ungdomar. 2013. Retrieved from http://www.internetmedicin.se/page. aspx?id=1087. Accessed February 21, 2017.

33. Klein ER, Armstrong SL, Shipon-Blum E. Assessing spoken language competence in children with selective mutism: using parents as test presenters. Commun Disord Quarter. 2013;34(3):184-195.

34. Gillberg C. The ESSENCE in child psychiatry: Early Symptomatic Syndromes Eliciting Neurodevelopmental Clinical Examinations. Res Dev Disabil. 2010;31(6):1543-1551.
Neuropsychiatric Disease and Treatment

\section{Publish your work in this journal}

Neuropsychiatric Disease and Treatment is an international, peerreviewed journal of clinical therapeutics and pharmacology focusing on concise rapid reporting of clinical or pre-clinical studies on a range of neuropsychiatric and neurological disorders. This journal is indexed on PubMed Central, the 'PsycINFO' database and CAS,

\section{Dovepress}

and is the official journal of The International Neuropsychiatric Association (INA). The manuscript management system is completely online and includes a very quick and fair peer-review system, which is all easy to use. Visit http://www.dovepress.com/testimonials.php to read real quotes from published authors.

Submit your manuscript here: http://www.dovepress.com/neuropsychiatric-disease-and-treatment-journal 